\title{
NRC and FDA Regulations Affecting Nuclear Pharmacy Practice
}

\author{
Neil A. Petry
}

Radiopharmaceuticals are radioactive drugs that are used in nuclear medicine practice for diagnosis and treatment of disease. Nuclear pharmacy practice is a patient-oriented pharmaceutical service that promotes the safe and efficacious use of radiopharmaceuticals and other drugs used in nuclear medicine, and is regulated jointly by a variety of federal, state. and local agencies. Given that the medical use of radiopharmaceuticals is highly regulated at multiple levels of government, it is not surprising that nuclear

\section{THE MEDICAL USE OF RADIOPHARMACEUTICALS}

$\mathbf{R}$ ADIOPHARMACEUTICALS are radioactive prescription drug products that are internally administered and intended for use in the diagnosis and treatment of disease. The term radiopharmaceutical also applies to nonradioactive reagent kits and radionuclide generators intended for use in the preparation of radioactive drugs and radioactive biological drug products; however, it does not include substances that contain trace quantities of naturally occurring radionuclides or sealed radionuclide sources.

The radiopharmaceuticals used in nuclear medicine are procured from commercial pharmaceutical companies that specialize in the manufacture and distribution of radioactive dosage forms. The majority of these radiopharmaceuticals are small volume parenterals, although oral solutions, capsules, aerosols, and gases are also used. Several of these radiopharmaceuticals are provided as final dosage forms ready for patient administration; however, the majority must be prepared (ie, compounded) just before patient administration due to their short physical halflife (ie, rapid radioactive decay) and in some cases limited chemical stability. Radiopharmaceuticals are typically prepared by nuclear pharmacists who practice in hospital pharmacies and/or nuclear medicine departments or within community-based centralized nuclear pharmacies. The majority of nuclear pharmacists practice in the centralized nuclear pharmacies that are generally located in large cities. These centralized nuclear pharmacies provide service to the majority of the hospitals and private nuclear medicine clinics located within each city and surrounding metropolitan or geographic area. pharmacy practice is conducted in a complex regulatory environment. The purpose of this article is to provide a brief overview of this regulatory environment, primarily in terms of two important regulatory agencies involved (Nuclear Regulatory Commission and Food and Drug Administration), their primary authority and activities, and the regulations that must be satisfied in order to assure the safe and efficacious use of radiopharmaceuticals.

(-) 1989 by W.B. Saunders Company.

Although nuclear pharmacy services are widely available, it is not uncommon to find that the basic pharmacy function is served by nuclear medicine technologists under the direct supervision of a qualified nuclear medicine physician. This arrangement is permissible in any clinical setting as it constitutes the practice of medicine. However, it is important to note that the hospital pharmacist must be aware of his or her responsibility to monitor the use of all drugs within the hospital including those used in nuclear medicine.

\section{NUCLEAR PHARMACY PRACTICE STANDARDS}

Nuclear pharmacy practice standards ${ }^{1}$ have been developed to supplement competency-based pharmacy practice standards and to identify those areas of responsibility that are unique to nuclear pharmacy. The standards function as guidelines for nuclear pharmacy practitioners and also provide the practice-oriented foundation upon which a competency-based certification exam is derived. These standards may also be useful to nuclear medicine physicians and technologists involved in the medical use of radiopharmaceuticals; however, the standards are not intended to govern the activities of these individuals. In addition, it is important to realize that many of these standards of practice are derived from existing regulations governing the medical use of

From the Division of Nuclear Medicine. Department of Internal Medicine. The University of Michigan Medical Center, Ann Arbor, $M I$.

Address reprint requests to Neil A. Petry. MS, RPh, BCNP, Nuclear Pharmacy, University Hospital B1G412/ 0028. The University of Michigan Medical Center, $1500 \mathrm{E}$ Medical Center Dr, Ann Arbor, MI 40109-0028.

○ 1989 by W.B. Saunders Company.

0897-1900/89/0205-000955.00/0 
radioactive materials; thus, there may be a legal requirement to comply with the described activities.

Nuclear pharmacy practice is a basic patientoriented pharmaceutical service that embodies the scientific knowledge and professional judgment required to improve and promote health through assurance of the safe and efficacious use of radioactive drugs for diagnosis and therapy. ${ }^{1}$ It is important to note that nuclear pharmacists practice in a variety of settings; therefore, their responsibilities may vary significantly in individual practice. The general areas of responsibility that comprise the practice of nuclear pharmacy include, but are not limited to, the procurement, compounding, quality control, dispensing, and distribution of radiopharmaceutical and pharmaceutical drug products used in nuclear medicine. In addition to these services, nuclear pharmacists

\section{It is important to note that the hospital pharmacist must be aware of his or her responsibility to monitor the use of all drugs within the hospital including those used in nuclear medicine.}

supervise supportive personnel, act as consultants and drug information specialists, conduct pharmaceutical and clinical research, act as radiation safety officers, and manage the complex regulatory affairs associated with the medical use of radiopharmaceuticals.

\section{REGULATORY HISTORY OF RADIOPHARMACEUTICALS}

The regulatory history of radiopharmaceuticals is fairly complex and is marked by several well documented milestones. The first significant regulatory event occurred with the enactment of the 1938 Food, Drug, and Cosmetic (FD\&C) Act, which for the first time required that the safety of all new drugs be proved to the Food and Drug Administration (FDA) before marketing. Approximately 6 years later, the Public Health Services Act of 1944 authorized the Bureau of
Biologics of the FDA to regulate radioactive biological drug products. During the formative years of nuclear medicine, radiopharmaceuticals were chiefly controlled by the Atomic Energy Commission $(\mathrm{AEC})^{2}$ as they contained byproduct radionuclides (ie, radioactive materials produced in nuclear reactors). The 1954 Atomic Energy Act authorized the AEC to license the possession, use, and transfer of by-product material, including radiopharmaceuticals. The enactment of the Kefauver-Harris Drug Amendments of 1962 increased federal control over the methods of production and testing of drugs before their release for sale to the public, and consequently threatened the availability of radiopharmaceuticals. This problem was solved in $1963^{3}$ when the FDA allowed a temporary exemption for radioactive new drugs and biologicals from the investigational new drug (IND) regulations, provided they were distributed in complete compliance with existing AEC regulations. The primary purpose of the temporary exemption was to allow the continued availability of radioactive drugs, manufactured from reactor-produced radionuclides, until such time that the FDA and AEC reached agreement regarding effective regulations that would minimize unnecessary duplication of regulatory control. The temporary exemption was rescinded, in part, on November 3, 1971, when the FDA actively entered the regulatory arena with the publication of new drug application (NDA) requirements for radioactive drugs. ${ }^{4}$ The new regulations identified specific radiopharmaceuticals that were no longer exempt from the new drug regulations because they were considered to be well established in nuclear medicine practice. Both the AEC and FDA concluded that it was inappropriate for these radiopharmaceuticals to be distributed under the investigational new drug exemption when they were intended for routine clinical use. ${ }^{4}$

During the transition period that followed, radiopharmaceutical manufacturers were allowed to distribute only those radioactive drug products for which an NDA, biological product license, or an IND was approved by the FDA. As a result of these regulatory requirements, 52 radiopharmaceutical NDAs were submitted and approved from 1971 through 1975 versus only 31 from 1951 through $1970 .^{5}$ On July 25 , 1975, the FDA issued a final rule ${ }^{6}$ which totally revoked 
the investigational new drug exemption and placed radiopharmaceuticals fully under the FDA's authority as are all other drug products. The penultimate regulatory milestone was reached on January 19, 1975 following the passage of the Energy Reorganization Act of 1974. The act abolished the AEC and created the Nuclear Regulatory Commission (NRC) which is now responsible for all licensing and regulatory functions originally assigned to the AEC by the Atomic Energy Act of 1954 (as amended).

\section{THE CURRENT REGULATORY ENVIRONMENT}

The medical use of radiopharmaceuticals is currently regulated by a variety of federal, state, and local agencies, which bear responsibility for assuring the general public that radiopharmaceuticals are used in a safe and efficacious manner. Recently, Vandergrift ${ }^{7}$ used a diagram to show the relationship between a nuclear medicine facility and various federal and state regulatory agencies. This diagram is useful; however, it does not fully describe the current regulatory environment as it fails to reflect the involvement of boards of medicine and pharmacy at the state level. Figure 1 more clearly portrays the current regulatory environment in which radiopharmaceuticals are used and reveals the regulatory relationships that exist between the medical use of radiopharmaceuticals and various regulatory agencies. As indicated, the NRC or an agreement state agency has primary and general authority over the medical use of radiopharmaceuticals in a given locale. State boards of medicine and pharmacy have only general authority in that they license individual practitioners who, with appropriate training and experience, may obtain NRC or agreement state agency authorization for the medical use of radiopharmaceuti-

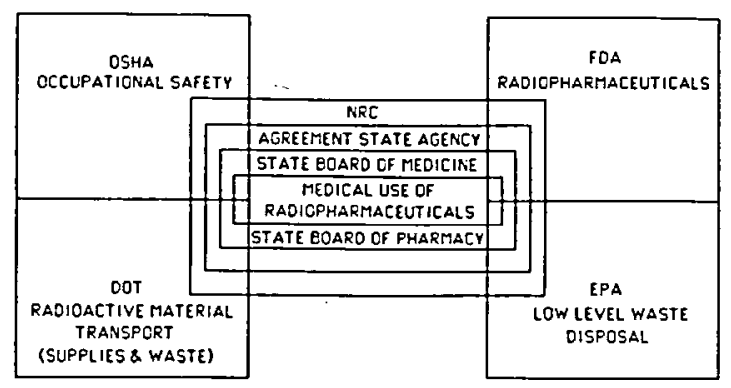

Fig 1. The regulatory environment associated with the medical use of radiopharmaceuticals. cals. The FDA and Department of Transportation have a moderate degree of regulatory authority while the Environmental Protection Agency and Occupational Safety and Health Administration have only limited authority.

It is important to realize that all of the identified agencies have significant responsibilities beyond those related to the regulation of the medical use of radiopharmaceuticals. For example, the NRC is also responsible for regulating the industrial use of nuclear reactor by-product materials as well as the fissionable materials used in the production of electrical power. In a similar fashion, the FDA maintains responsibility for the research and development of safe and effective drugs, biological products, and medical devices and their marketing, distribution, and clinical use. A detailed discussion of all regulatory agencies involved in the regulation of the medical use of radiopharmaceuticals is beyond the intended scope of this article; therefore, the reader is referred elsewhere for additional detail. ${ }^{8,9}$ The remaining discussion will be limited to a brief overview of the regulatory authority and activities of the NRC and FDA since the regulations of these federal agencies have the greatest impact on nuclear pharmacy practice.

\section{REGULATORY AUTHORITY OF THE NRC AND THE FDA}

Following the revocation of the investigational new drug exemption for radiopharmaceuticals, the FDA began to regulate actively the safety and efficacy of radiopharmaceuticals with respect to patients. In response, the NRC withdrew from regulating the safety and efficacy of radiopharmaceuticals and began to focus its regulatory activities on the safety of occupational workers and the general public. In 1979, the NRC published a policy statement to guide its regulation of the medical use of radiopharmaceuticals. ${ }^{10}$ In this three-part policy statement, the NRC indicated that it would: (1) continue to regulate the medical use of radioisotopes as necessary to provide for the radiation safety of workers and the general public; (2) regulate the radiation safety where justified by the risk to patients and where voluntary standards or compliance with these standards is inadequate; and (3) minimize intrusion into medical judgments affecting patients and into other 
areas traditionally considered to be part of the practice of medicine. It is also important to note that the policy statement indicated that "the NRC intends not to exercise regulatory control in those areas (involving patients) where, upon careful examination, it determines that there are adequate regulations by other Federal or State agencies or well administered standards. The Commission recognizes that the FDA regulates the manufacture, interstate distribution, investigational and research use of drugs, including radiopharmaceuticals, but does not have the authority to restrict the routine use of drugs to the procedures (specified in the product labeling) that FDA has approved as safe and effective. The NRC sees itself as the only Federal Agency that is currently authorized to regulate the routine use of radioactive drugs from the standpoint of reducing unnecessary radiation exposure to patients,"10

\section{THE NRC AND NUCLEAR PHARMACY PRACTICE}

The NRC has legislative authority to license and regulate all aspects of the possession, use, and disposal of by-product materials in order to protect health and minimize danger to life and property. Thus, it also has certain regulatory authority over radiopharmaceuticals. This authority does not, however, include the regulation of radiopharmaceuticals that contain naturally occurring or accelerator-produced radionuclides as they are regulated at the state level. The NRC has agreed to transfer authority to control the use of reactor-produced materials to 29 state regulatory agencies. Under the current regulatory scheme, the NRC regulates reactor-produced materials only, the nonagreement states regulate accelerator-produced materials only, and the agreement states regulate all radioactive materials.
All NRC regulations are published in the Federal Register and codified in Title 10, Chapter 1, of the Code of Federal Regulations (CFR). The NRC regulations pertaining to the medical use of by-product materials are identified in NRC Regulatory Guide $10.8^{11}$ and listed in Table 1 . The regulations that most directly affect nuclear pharmacy practice are contained in 10 CFR 35 (Human Uses of By-product Material), 10 CFR 20 (Standards for Protection Against Radiation), and 10 CFR 71 (Packaging and Transportation of Radioactive Materials). The remaining regulations are equally important in that nuclear pharmacies must operate in total compliance with applicable sections of each part; however, these regulations are primarily administrative in nature and do not directly impact on routine practice activities. Therefore, the following discussion will focus on selected sections of 10 CFR 35 in an effort to characterize the regulatory relationship that exists between the NRC and nuclear pharmacy practice.

The regulations contained in 10 CFR 35 prescribe requirements and provisions for the medical use of by-product material, the issuance of specific licenses authorizing the medical use of this material, and the protection of the public health and safety. Given that these are federal regulations, it is not surprising to find that they serve as part of the foundation upon which the nuclear pharmacy practice standards ${ }^{1}$ are based. This relationship is clearly identified in the practice standards in that nuclear pharmacists have a professional responsibility to comply with the NRC and/or state license(s) under which the nuclear pharmacy operates, and federal, state and institutional rules regulating radiation and radiopharmaceuticals. More specifically, 10 CFR 35.11 specifies that a person shall not manufac-

Table 1. United States Nuclear Regulatory Commission Rules and Regulations Pertaining to the Medical Use of By-Product Materials

\begin{tabular}{|c|c|c|}
\hline 10 CFR Part & 19 & Notices, instructions, and reports to workers; inspections \\
\hline 10 CFR Part & 20 & Standards for protection against radiation \\
\hline 10 CFR Part & 21 & Reporting of defects and noncompliance \\
\hline 10 CFR Part & 30 & $\begin{array}{l}\text { Rules of general applicability to domestic licensing of by-product } \\
\text { material }\end{array}$ \\
\hline 10 CFR Part & 35 & Medical use of by-product material \\
\hline 10 CFR Part & 71 & Packaging and transportation of radioactive material \\
\hline 10 CFR Part & 170 & $\begin{array}{l}\text { Fees for facility and materials and other regulatory services under } \\
\text { the Atomic Energy Act of 1954, as amended }\end{array}$ \\
\hline
\end{tabular}


ture, produce, acquire, receive, possess, use, or transfer by-product material for medical use except in accordance with a specific license issued by the NRC or an agreement state, which authorizes these activities. This licensure requirement is reflected in the procurement practice standards which obligate nuclear pharmacists to act with proper authority (ie, under a valid radioactive materials license) to purchase radioactive material.

Title 10 CFR 35.20 specifies that each licensee shall develop and implement a written radiation protection program that includes provisions for keeping radiation exposure of employees, patients, visitors, and the public as low as reasonably achievable (ALARA). The ALARA concept is embraced throughout the practice

\section{The ALARA concept is embraced throughout the practice standards as nuclear pharmacists are obligated to work jointly with the radiation safety officer and/or health physicist and nuclear medicine physician in developing radiation protection procedures that comply with this legal standard.}

standards as nuclear pharmacists are obligated to work jointly with the radiation safety officer and/or health physicist and nuclear medicine physician in developing radiation protection procedures that comply with this legal standard. Numerous radiation protection procedures, such as the use of time, distance, and shielding techniques, are prescribed in the practice standards, and additionally many of these procedures are specifically required by regulation. For example, 10 CFR 35.60 specifies that a licensee shall require each individual who prepares (ie, compounds) a radiopharmaceutical kit to use a syringe radiation shield and shall also require individuals to use a syringe radiation shield when administering a radiopharmaceutical by injection unless the use of the shield is contraindicated for that patient. This regulation also requires each syringe or syringe radiation shield that contains a syringe with a radiopharmaceutical to be conspicuously labeled so that the contents may be identified; however, this requirement is limited to providing the radiopharmaceutical name or its abbreviation, the clinical procedure to be performed, or the patient's name. In this regard it is interesting to note that nuclear pharmacists are obligated, under both federal and state laws and pharmacy practice standards, to provide for each radiopharmaceutical dosage an appropriate prescription label containing all legally required information. ${ }^{12}$ In addition to this required information, radiopharmaceutical prescription labels must include the amount of radioactivity, the calibration and expiration time, and a cautionary statement and symbol indicating that the dosage is radioactive in order to further assure that the dosage is used in a safe and efficacious manner.

In some instances conflict may exist between NRC regulations and the standards of nuclear pharmacy practice. For example, 10 CFR 35.200 specifies that a licensee may use any by-product material in a diagnostic radiopharmaceutical or any generator or reagent kit for the preparation and diagnostic use of a radiopharmaceutical containing by-product material for which the FDA has accepted a IND or approved a NDA. The regulation also specifies that a licensee shall elute generators and prepare reagent kits in accordance with the manufacturer's instructions. This latter requirement is seldom a problem as nuclear pharmacists most frequently adhere to the manufacturer's instructions. However, as professional practitioners, nuclear pharmacists must reserve the right to exercise professional judgment as appropriate to the provision of quality nuclear pharmacy services and the overall safety of nuclear medicine procedures. Thus, based upon a physician's prescription, patient needs, and individual experience with the prescribed radiopharmaceutical, nuclear pharmacists may modify the manufacturer's instructions or in some cases develop compounding procedures that are not described by the manufacturer. As an example, nuclear pharmacists may establish specialized procedures for preparing pediatric doses of technetium ${ }^{99 \mathrm{~m}} \mathrm{Tc}$ Albumin Aggregated ( ${ }^{99 m}$ Tc MAA) for pulmonary perfu- 
sion studies ${ }^{13}$ as the instructions for preparing the radiopharmaceutical for pediatric patients in a reasonable volume of administration are not provided in the product labeling. Obviously, nuclear pharmacists must consider the legal ramifications associated with this and other practice activities that may deviate from applicable NRC regulations.

\section{THE FDA AND NUCLEAR PHARMACY PRACTICE}

The FDA is a scientific federal regulatory agency charged with the responsibility for ensuring that: (1) foods are safe, pure, and wholesome; (2) cosmetics are safe; (3) drugs, biological products, and medical devices are safe and effective; (4) radiologic products and their uses do not result in unnecessary radiation exposure; and (5) all these products are properly and honestly labeled. ${ }^{14}$ Consequently, the FDA's regulatory activities have a significant affect on the medical use of radiopharmaceuticals and nuclear pharmacy practice. The regulatory relationship that currently exists between the FDA and nuclear pharmacy practice has been described in a detailed policy statement ${ }^{15}$ and will be considered briefly here.

During the formative years, nuclear pharmacy practice activities included: (1) purchasing a commercially prepared radiopharmaceutical from a drug manufacturer who held an approved NDA and dispensing the drug in its original unopened container; (2) dispensing a single patient dose from a multiple dose container of a commercially prepared radiopharmaceutical; and (3) diluting (including adjustments of buffers, bacteriostatic agents, and stabilizers) and repackaging a commercially prepared radiopharmaceutical for subsequent use or distribution. Eventually, as technology developed and practitioners gained more training and experience, nuclear pharmacies expanded their activities to include: (1) eluting a NDA approved radionuclide generator and using the eluate to compound a radiopharmaceutical from a nonradioactive reagent kit; (2) preparing a nonradioactive reagent kit for subsequent use in compounding a radiopharmaceutical; and (3) preparing a radiopharmaceutical by using a commercially prepared radionuclide or a radionuclide obtained from a local nuclear reactor or cyclotron. As these compounding and distribution became more prevalent, it became evident that even though most nuclear pharmacy activities clearly involved pharmacy practice, others constituted manufacturing which required the nuclear pharmacy to register as a drug establishment under Section 510 of the FD\&C Act. Consequently, nuclear pharmacies that are required to register are subject to the drug listing provisions of Section 510 of the FD\&C Act and FDA's regulations under 21 CFR 207, the current good manufacturing practice requirements of Section 501 of the FD\&C Act and FDA's regulations under 21 CFR 210 and 211 , and the factory inspection provisions of Section 505 of the FD\&C Act and FDA's regulations under $21 \mathrm{CFR} 310,312$, and 314.

As nuclear pharmacy practice matured further, a controversy developed between practitioners and the FDA regarding what nuclear pharmacy activities should be considered as the practice of pharmacy and what activities should be considered as manufacturing. To aid the FDA in establishing criteria to determine what nuclear pharmacy activities required registration, a subcommittee of the FDA's Radiopharmaceuticals Advisory Committee was appointed in October 1975 to consider the existing issues. The FDA specifically requested that the subcommittee consider: (1) the types of operations engaged in by nuclear pharmacies that should be regulated to some degree by FDA; and (2) the relative urgency for issuing new regulations or statements of policy, if they were needed.

On April 15, 1976, the subcommittee reported to the Radiopharmaceuticals Advisory Committee. In considering which nuclear pharmacy operations should be regulated by the FDA, the subcommittee concluded that: (1) if the radiopharmaceutical was prepared and dispensed under a prescription, the laws and regulations governing the practice of pharmacy and medicine at the state level should apply and the nuclear pharmacy should be considered as engaging in the practice of pharmacy; and (2) the presence of a third party in the distribution of a prescription drug, between the location where the product is formulated, compounded, or manufactured and the point where it is administered to patients, changes the practice to one of manufacturing. Examples of the latter situation include one in which a nuclear pharmacy sells radiopharmaceuticals to a second pharmacy for dispensing 
pursuant to a prescription, and a second in which a nuclear pharmacy sells to other pharmacies bulk quantities of nonradioactive reagent kits that it has developed. In each case the first pharmacy would be a manufacturer under the FD\&C Act and be required to register under Section 510 of the Act.

The subcommittee also recognized that radiopharmaceuticals are frequently administered by the nuclear medicine unit in a single institution. These units may operate a nuclear pharmacy and maintain control over any radiopharmaceutical manufactured or compounded within the pharmacy until it is dispensed for patient administration. Because of the high level of control exercised over the drugs in this setting, the subcommittee concluded that there was no need

\section{If the radiopharmaceutical was \\ prepared and dispensed under a prescription, the laws and regulations governing the practice of pharmacy and medicine at the state level should apply and the nuclear pharmacy should be considered as engaging in the practice of pharmacy}

for registration. In addition, the subcommittee recommended that: (1) substantive changes in FDA regulations, as they pertain to true nuclear pharmacies, were not needed; (2) individual state boards of pharmacy rather than the FDA should regulate nuclear pharmacies in the same manner as they regulate traditional pharmacies that do not compound or dispense radiopharmaceuticals; and (3) the FDA should issue a policy statement to clarify the issue of nuclear pharmacy manufacturing $v$ traditional pharmacy compounding.

In response to the subcommittee's recommendations, the FDA responded with a policy statement ${ }^{15}$ to guide its regulation of the activities associated with nuclear pharmacy practice. In this policy statement, the FDA noted that Section $510(\mathrm{~g})(1)$ of the FD\&C Act [21 USC $360(\mathrm{~g})(1)]$ states that "pharmacies which maintain establishments in conformance with any applicable local laws regulating the practice of pharmacy and medicine are exempt from the drug registration provisions of the Act. For the exemption to apply they must be regularly engaged in dispensing prescription drugs or devices, upon prescriptions of practitioners licensed to administer such drugs or devices to patients under the care of such practitioners in the course of their professional practice, and they must not manufacture, prepare, propagate, compound, or process drugs or devices for sale other than in the regular course of their business of dispensing or selling drugs or devices at retail."15

The FDA, however, does not believe that this pharmacy exemption applies to all nuclear pharmacies, because many states do not appear to have laws that apply specifically to the practice of nuclear pharmacy. Also, many states may not have mechanisms specifically designed for certifying nuclear pharmacists, and many nuclear pharmacies require specifically trained personnel whose activities resemble those of drug manufacturers more than those of traditional pharmacy practice. Consequently, the FDA's regulation of nuclear pharmacies will be based on reasonable application of the provisions of Section 510 (g)(1) of the FD\&C Act to nuclear pharmacies and it will treat these nuclear pharmacies as other pharmacies have been traditionally treated under this section. Therefore, a nuclear pharmacy whose activities are consistent with Section $510(\mathrm{~g})(1)$ of the FD\&C Act will be exempt from registering as a drug establishment and from complying with other requirements under the section. Conversely, a nuclear pharmacy whose activities fall outside the provisions of Section $510(\mathrm{~g})(1)$ of the FD\&C Act will be subject to the registration provisions and to those requirements that are concomitant to registration. The FDA has provided several specific examples of cases when a nuclear pharmacy must register as a drug establishment. ${ }^{15}$

\section{SUMMARY}

In conclusion, all pharmacists should have a basic understanding of the regulations governing the medical use of radiopharmaceuticals and the practice of nuclear pharmacy. Federal regula- 
tions promulgated by the NRC and FDA are most important; however, state and local regulations must also be given due consideration. NRC regulations focus primarily on providing for the radiation safety of workers and the general public while FDA regulations focus on the safety and efficacy of radiopharmaceuticals with respect to patients. Nuclear pharmacists bear responsibility for assuring that these regulations are satisfied and for providing the clinical pharmacy services that are required to assure the safe and efficacious use of radiopharmaceuticals.

\section{REFERENCES}

1. Nuclear Pharmacy Practice Standards: American Pharmaceutical Association, Washington, DC, 1984

2. Meyers EL: Radiopharmaceuticals: Regulatory problems, in Subramanian G, Rhodes BA, Cooper J, et al (eds): Radiopharmaceuticals. New York, NY, Society of Nuclear Medicine, 1975, pp 259-263

3. Food and Drug Administration: Radioactive new drugs for investigational use. Fed Reg 28:183, January 8, 1963

4. Food and Drug Administration: Radioactive new drugs: New-drug application requirements. Fed Reg 36: 21026, November 3, 1971

5. Halperin JA, Stringer SA, Eastep RD: Regulation of radiopharmaceuticals: Analysis of radiopharmaceuticals approved for marketing in the United States 1951-1978, in: Radiopharmaceuticals II, Proceedings of the Second International Symposium on Radiopharmaceuticals. New York, NY, Society of Nuclear Medicine, 1979, p xxi

6. Food and Drug Administration: Radioactive new drugs and radioactive biologics. Termination of exemptions. Fed Reg 40:31298, July 25, 1975

7. Vandergrift, JF: Regulatory problems in nuclear medicine, in Hladik WB, Saha GB, Study KT (eds): Essentials of Nuclear Medicine Science. Baltimore, MD, Williams \& Wilkins, 1987, pp 321-330
8. Kowalsky RJ, Perry JR: Licensing, regulatory control and radiation safety, in: Radiopharmaceuticals in Nuclear Medicine Practice. Norwalk, CT, Appleton \& Lange, 1987, pp 473-500

9. Chilton HM, Witcofski RL: Regulations affecting radiopharmaceuticals, in: Nuclear Pharmacy: An Introduction to the Clinical Application of Radiopharmaceuticals. Philadelphia, PA, Lea \& Febiger, 1986, pp 150-157

10. Nuclear Regulatory Commission: Regulation of medical uses of radioisotopes. Statement of general policy. Fed Reg 44:8242, February 9, 1979

11. Guide for the preparation of applications for medical use programs: Regulatory Guide 10.8 (Task FC 415-4), Revision 2. Washington, DC, Nuclear Regulatory Commission, August 1987

12. Fink JL, Marquardt KW, Simonsmeier IM (eds): Pharmacy Law Digest, Facts and Comparisons. Philadelphia, PA, Lippincott, 1987, pp DC-19

13. Levine G, Massetti C, Malhi B: A methodology for preparing pediatric doses of ${ }^{99 \mathrm{~m}} \mathrm{Tc}$ MAA for pulmonary perfusion studies. J Nucl Med Technol 8:94.96, 1980

14. Siegel BA: Radiopharmaceuticals and FDA: A clinician's perspective. J Nucl Med Technol 11:177-186, 1983

15. Nuclear Pharmacy Guideline: Criteria for determining when to register as a drug establishment, Division of Drug Labeling and Compliance (HFN-310). Washington, DC, Center for Drugs and Biologics, Food \& Drug Administration, May 1984 